\title{
Prostatectomía radical laparoscópica: la curva de aprendizaje con tutor
}

\author{
Raventós Busquets CX, Cecchini Rosell L, Trilla Herrera E, Planas Morin J, \\ Orsola de los Santos A, Morote Robles J.
}

Servicio de Urología. Hospital General Universitari Vall d'Hebron. Barcelona.

Actas Urol Esp. 2007:31(3);205-210

\section{RESUMEN}

PROSTATECTOMÍA RADICAL LAPAROSCOPICA: LA CURVA DE APRENDIZAJE CON TUTOR

Objetivos: Exponer con detalle el sistema de aprendizaje de la prostatectomía radical laparoscópica (PRL) con tutor llevado a cabo en nuestro centro así como los resultados.

Material y métodos: Se realiza el análisis de variables intraquirúrgicas, postoperatorias y del control quirúrgico y oncológico de la pieza, de 105 PRL (marzo 2004-diciembre 2005) según los siguientes grupos: Grupo 1: tutor como primer cirujano y alumno como asistente. Grupo 2 alumno como primer cirujano y el tutor como ayudante. Grupo 3: alumno como primer cirujano y otro alumno/residente como ayudante.

Resultados: No se han observado diferencias significativas en cuanto la media de tiempo quirúrgico (TQ) entre el grupo 2 y 3 (188'-170', p=0,09). Ésta ha sido menor en el Grupo 1 (150', p<0,05), disminuyendo con el tiempo. La pérdida sanguínea, el control quirúrgico-oncológico, el estadio patológico y el tiempo de estancia hospitalaria han sido similares en los tres grupos.

Conclusiones: La presencia de un laparoscopista experto como tutor permite aprender la PRL de manera segura y efectiva. En espera de los resultados funcionales y oncológicos a largo plazo, este sistema de aprendizaje no se asocia ni con un mayor riesgo para el paciente ni con un peor control quirúrgico/oncológico. Consideramos que este método es fácilmente reproducible y permite un acortamiento de la curva de aprendizaje.

Palabras clave: Prostatectomía radical laparoscópica. Curva de aprendizaje. Cáncer de próstata.

\section{ABSTRACT}

LAPAROSCOPIC RADICAL PROSTATECTOMY: LEARNING CURVE WITH A MENTOR TRAINING PROGRAM

Introduction: We describe and evaluate the results of our mentor training program for laparoscopic radical Prostatectomy (LRP).

Material and Methods: From March 2004 through December 2005, we have performed 105 (LRP). Three groups have been analysed: Group 1: The mentor as the first surgeon with the trainee acting as the assistant. Group 2: The trainee as the first surgeon with the mentor acting as the assistant. Group 3: The trainee as the first surgeon with another trainee/resident as the assistant. We have evaluated operative, postoperative data and surgical/oncological control.

Results: There was no statistical difference in mean operative time in Groups 2 and 3 (200'-198'), but there was a difference from Group $1\left(148,4^{\prime}\right)(\mathrm{p}<0,05)$ we have observed a progressive operative time decrease only in Group 1. Blood loss, surgical-oncological control, pathological stage and hospital stay have been similar in the three groups.

Conclusions: Skills for LRP can be effectively and safely taught by the presence of an experienced mentor. Waiting for long term results according to potency and continence, it was not associated to higher patient risk, neither to a worse surgical/oncologic outcome. We consider that this program is reproducible and allows a shorter learning curve.

Keywords: Laparoscopic radical prostatectomy. Learning curve. Prostate cancer. 
$\mathrm{L}^{2}$ a prostatectomía radical laparoscópica (PRL) se esta introduciendo en nuestro entorno como una nueva técnica en la cirugía del cáncer de próstata. La curva de aprendizaje es larga y difícil para los cirujanos sin experiencia en procedimientos laparoscópicos. En estos momentos se puede hablar de una segunda generación de urólogos laparoscopistas que se está iniciando y se benefician de la primera, con una curva de aprendizaje más corta (Fig. 1). Exponemos la experiencia de nuestro centro con detalles del sistema de aprendizaje así como los resultados.

\section{MATERIAL Y MÉTODO}

El Servicio empezó la cirugía laparoscópica en marzo del 2004 al incorporar la figura de un tutor (LL.C.). Éste había realizado hasta entonces aproximadamente un total de cincuenta casos de prostatectomía radical, veinte nefrectomías y diez cistectomías radicales laparoscópicas.

Desde su incorporación hasta enero del 2006 (22 meses) se han realizado en nuestro centro un total de 180 prostatectomías radicales, 105 de las cuales han sido por laparoscopia (20 por la vía extraperitoneal).

\section{Grupos}

Se ha estudiado la evolución en la cirugía entre: los alumnos y el urólogo laparoscopista con experiencia, y entre los mismos alumnos. El primer año se iniciaron dos alumnos y a lo largo de 2005 se incorporaron 3 urólogos más, siendo un total de 5 alumnos. Todos ellos habían realizado previamente programas de formación con animales y usan habitualmente el pelvi-trainer.

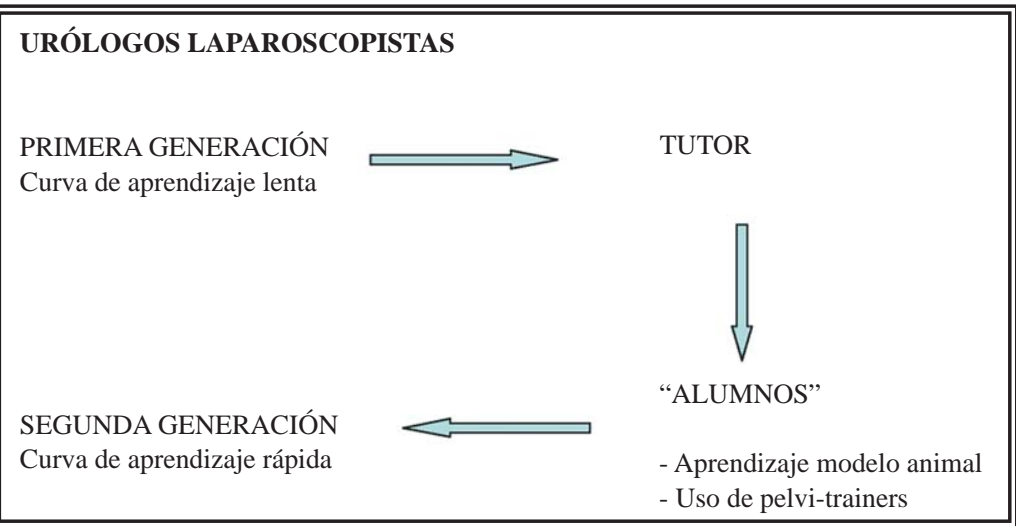

FIGURA 1. Esquema de la evolución del aprendizaje de la cirugía laparoscópica.
Se han evaluado los siguientes grupos:

- Grupo 1: tutor como primer cirujano y alumno como asistente (54 casos).

- Grupo 2 alumno como primer cirujano y el tutor como ayudante (18 casos).

- Grupo 3: alumno como primer cirujano y otro alumno/residente como ayudante (33 casos).

La Tabla 1 muestra la participación de cada alumno y de los residentes del Servicio en los diferentes grupos aprendizaje.

\section{Técnica quirúrgica}

En cuanto a la técnica quirúrgica se realizó inicialmente la descrita por el grupo de Montsouris ${ }^{1}$ con alguna modificación y obviando la disección de la vía seminal en el fondo de saco de Douglas.

Se coloca sonda vesical con una pequeña cantidad de globo (3-4 cc), seguidamente se colocan de los trócares según el esquema adjunto (Fig. 2). El primero con una incisión supraumbilical transperitoneal mediante el método de Hasson ${ }^{2}$. Se inicia bajo visión el acceso al espacio prevesical de Retzius mediante la sección del uraco y ligamentos umbilicales. Apertura del peritoneo parietal en dirección al orificio inguinal, siguiendo la línea de los ligamentos umbilicales. Disección roma hasta descubrir el pubis. Disección espacios latero-prostáticos. Cuándo no se pretende la conservación de los haces neurovasculares, se abre la fascia endopélvica. Limpieza-coagulación del la grasa preprostática y localización del cuello vesical.

Apertura lateral de la uretra prostática, esqueletizando el cuello, maniobra que se facilita deshinchando el globo de la sonda.

Coagulación y sección de la unión vesico-prostática antero-lateral. Sección vertical de la uretra a nivel del cuello vesical. Acceso vertical al plano vesicular a traves de las fibras que marcan la lámina anterior de la fascia de Denonvilliers. Localización y sección de los deferentes y liberación de ambas vesículas seminales. Apertura de la lámina posterior de la fascia de Denonvilliers para mejor control de los pedículos laterales y del recto. Para una mejor preservación de los haces 
Tabla 1

\begin{tabular}{lccccc}
\hline Cirujanos & Grupo 1 & Grupo 2 & Grupo 3 $1^{\circ}$ & Grupo 3 2o & Participación \\
\hline Tutor & 54 (20 extra) & 20 & - & - & 74 \\
Alumno 1 & $11(2)$ & 10 & 15 & 3 & 39 \\
Alumno 2 & $6(2)$ & 2 & 13 & 3 & 24 \\
Alumno 3 & $9(4)$ & 3 & 5 & 10 & 27 \\
Alumno 4 & $12(1)$ & 2 & 2 & 1 & 17 \\
Alumno 5 & $4(4)$ & 3 & 0 & 4 & 11 \\
Residente & 11 & 0 & 0 & 14 & 25
\end{tabular}

queidad. Colocación drenaje aspirativo "redón". Extracción de la pieza mediante bolsa por orificio trócar umbilical.

Cuando se realiza por vía extraperitoneal, la incisión inicial es infraumbilical y el acceso al espacio de Retzius se realiza mediante disección roma primero con el dedo $\mathrm{y}$ con la óptica después Se colocan el resto de los trócares en la misma posición.

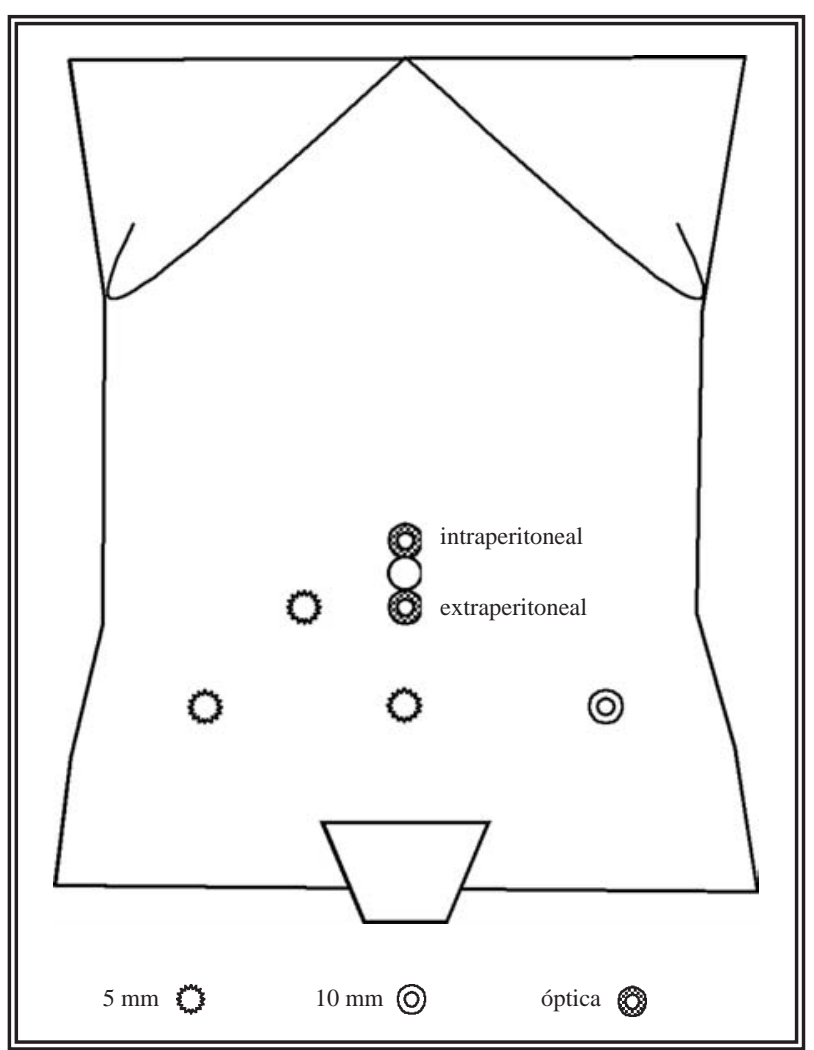

FIGURA 2. Colocación de los trócares en la prostatecto mía radical laparoscópica.

neurovasculares es mejor conservarla. Coagulación y sección de los pedículos laterales con mayor o menor márgen según cada caso. Coagulación/punto hemostático y sección del complejo de Santorini. Sección fría de la uretra a nivel del ápex prostático.

Exéresis de la pieza que se deja, durante el tiempo de la sutura de la anastomosis, a nivel del ciego/sigma. Anastomosis uretra-vesical con dos hemicicunferencias continuas de vicryl 3/0, que se anudan al final entre sí. Comprobación estan-
Se ha descrito para cada grupo las variables edad, volumen prostático, PSA y el estadio clínico y patológico. Asimismo, se han comparado las diferencias en cuanto al tiempo quirúrgico (TQ) en los tres grupos y la evolución del mismo a lo largo de los dos años.

También se ha analizado la pérdida sanguínea definida como la diferencia del valor de hematocrito pre y post quirúrgico y el tiempo de estancia hospitalaria. Finalmente, se ha comparado el control quirúrgico y la presencia de márgenes positivos (control oncológico). El control quirúrgico se define como la existencia de incisiones quirúrgicas sean con glándulas benignas en el borde del corte (IQB) o con cáncer en el mismo (IQM (malignas)). El control oncológico se define como la presencia márgenes positivos (Fig. 3).

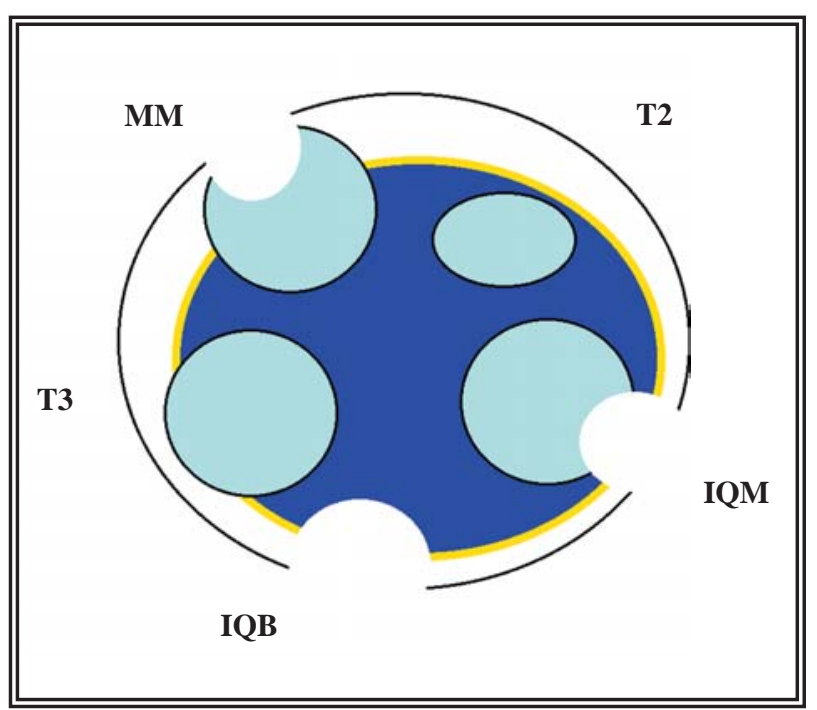

FIGURA 3. Dibujo de la glándula prostática donde se representa la Incisión Quirúrgica Benigna (IQB), la Incisión Quirúrgica Maligna (IQM) y los Márgenes Malignos (MM). 
Las diferencias entre variables cualitativas se han analizado mediante el test chi-cuadrado. Las diferencias entre variables cualitativas se han evaluado con el test de Fisher-Student.

\section{RESULTADOS}

La media de la edad, volumen prostático, PSA, el valor del Gleason y el estadio clínico no han diferido significativamente entre los tres grupos (Tabla 2). No se han observado diferencias significativas en cuanto la media de TQ entre el grupo 2 y 3 (200' y 198,8') (Tabla 3). Como era de esperar, la media de TQ ha sido menor en el Grupo 1 $\left(148,4^{\prime}\right)(p<0,05)$, observándose dentro de éste, una disminución progresiva significativa en el tiempo (Fig. 4).

Tabla 2

\begin{tabular}{lcccc}
\hline & Grupo I & Grupo II & Grupo III & Valor de p \\
\hline Edad $\pm \mathrm{DE}$ & $64,9 \pm 5,5$ & $65,1 \pm 6,4$ & $65,4 \pm 6,4$ & $\mathrm{p}>0,05$ \\
$\mathrm{PSA} \pm \mathrm{DE}$ & $7 \pm 2,2$ & $7,6 \pm 2,8$ & $6,9 \pm 2,3$ & $\mathrm{p}>0,05$ \\
Volumen $\pm \mathrm{DE}$ & $41 \pm 22,5$ & $41,6 \pm 18,1$ & $33,8 \pm 18,1$ & $\mathrm{p}>0,05$ \\
Gleason (\%) & & & & \\
$\leq 6$ & 48 & 55,6 & 51,5 & $\mathrm{p}>0,05$ \\
$\geq 7$ & 52 & 44,4 & 48,5 & \\
Estadio clínico(\%) & & & & \\
T1 & 67,9 & 88,9 & 75,8 & $\mathrm{p}>0,05$ \\
T2 & 33,1 & 11,1 & 24,2 & \\
\hline
\end{tabular}

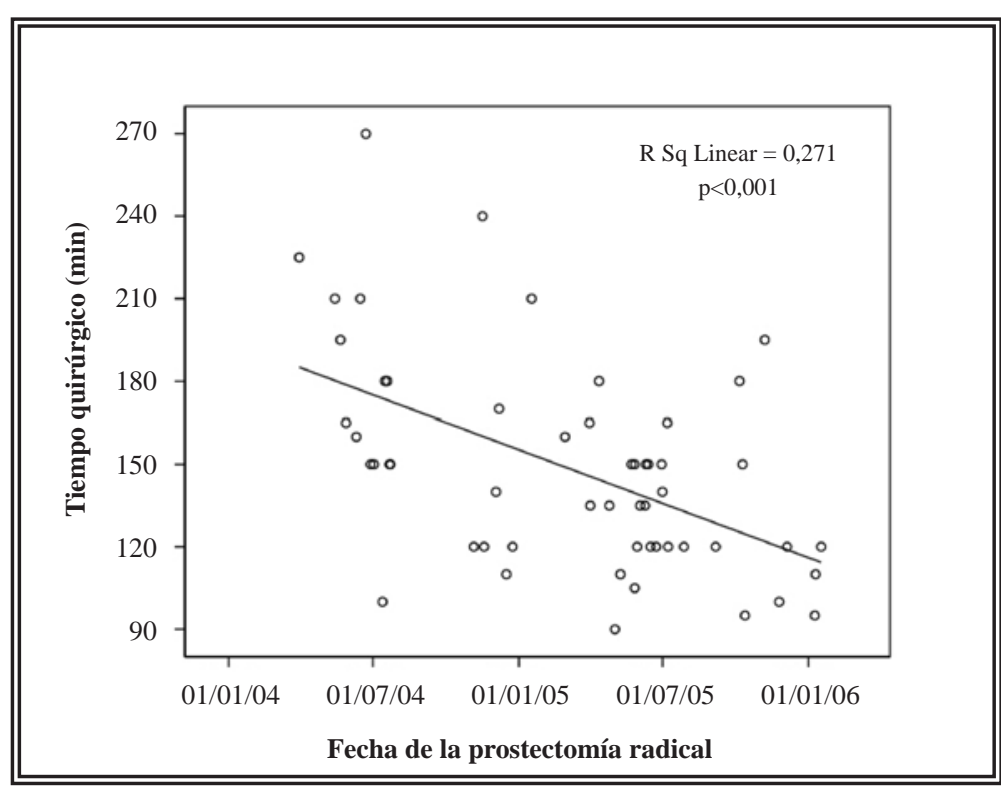

FIGURA 4. Evolución del tiempo quirúrgico en el Grupo 1.
Tabla 3

\begin{tabular}{lccc}
\hline & Grupo I & Grupo II & Grupo III \\
\hline $\begin{array}{l}\text { Tiempo } \\
\text { medio (min) }\end{array}$ & 148,4 & 200 & 198,8 \\
IC95\% & $137,3-159$ & $182,2-217,7$ & $187,6-210$ \\
Rango & & & \\
(máx-min) & $180(270-90)$ & $\begin{array}{c}140(320-180) \\
p<0,001\end{array}$ & $60(260-140)$ \\
& & $p<0,001$ & $p$ \\
& & & p ns \\
\hline
\end{tabular}

Aunque esta reducción del tiempo quirúrgico a lo largo de los 2 años no se observa en la cirugía realizada por los grupos 2 y 3 , éste empieza a disminuir por debajo de las tres horas en alguno de los últimos casos (Fig. 5). Tanto la pérdida sanguínea, el control quirúrgico-oncológico, el estadio patológico final y el tiempo de estancia hospitalaria han sido similares en los tres grupos. (Tablas 4 y 5).

\section{DISCUSIÓN}

El uso de la técnica laparoscópica está creciendo en los últimos años y la prostatectomía radical laparoscópica se considera como el procedimiento de más dificultad. El método más adecuado para el aprendizaje de dicha técnica es objeto de discusión en muchos foros. Se trata de un procedimiento difícil pero con la ventaja del elevado número de casos. Debido además a su menor riesgo de graves complicaciones/mortalidad intra-operatoria, algunos autores abogan que representa un modelo mejor que la nefrectomía ${ }^{3}$.

Creemos que existen dos aspectos básicos y complementarios en el aprendizaje:

- El primero relacionado con el aspecto de habilidad psicomotora como es la coordinación visión-manos en un espacio tridimensional con un monitor bidimensional.

- El segundo es la aplicación de esta habilidad ya adquirida en un paciente real.

El primer objetivo se consigue mediante la realización de cursos de laparoscopia, 


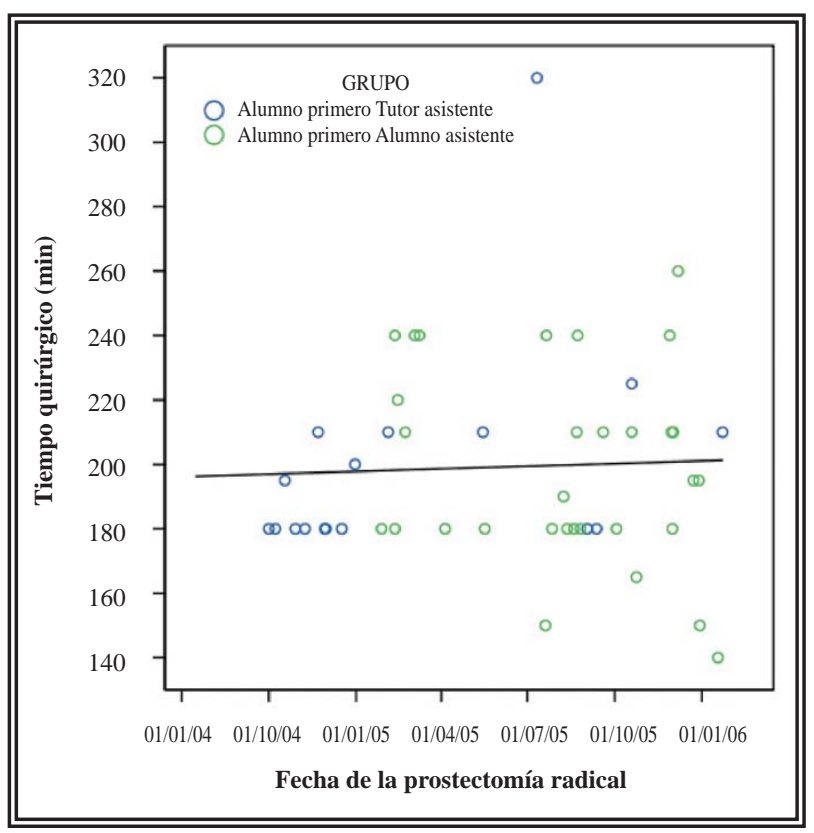

FIGURA 5. Evolución del tiempo quirúrgico en los grupos 2 y 3.

existiendo ya modelos para el entrenamiento de los residentes en las habilidades básicas, sin el estrés quirúrgico del paciente real. El pelvi-trainer ("dry-lab") es por tanto una herramienta útil para asimilar los movimientos y la coordinación más básicos como la colocación de la aguja en el porta. El modelo animal ("wet-lab") ofrece la posibilidad del aprendizaje de la disección de los tejidos y control hemostático de los vasos imposible en un pelvi-trainer.

Una vez sedimentadas estas habilidades, hay que iniciarse en la cirugía real. Creemos que la

Tabla 4

\begin{tabular}{lcccc}
\hline & Grupo I & Grupo II & Grupo III & Valor de p \\
\hline Diferencia Hto $\pm \mathrm{DE}$ & $9,01 \pm 3,74$ & $8,8 \pm 3,6$ & $9,7 \pm 5,7$ & $\mathrm{p}>0,05$ \\
Días ingreso $\pm \mathrm{DE}$ & $4,71 \pm 1,53$ & $4,7 \pm 1,19$ & $4,8 \pm 1,49$ & $\mathrm{p}>0,05$ \\
\hline
\end{tabular}

Tabla 5

\begin{tabular}{lccccc}
\hline & Grupo I & Grupo II & Grupo III & Valor de p & total \\
\hline $\begin{array}{l}\text { Estadio patologico } \\
\text { (T2/T3)(\%) }\end{array}$ & $80 / 20$ & $75 / 25$ & $88,2 / 11,8$ & $\mathrm{p}>0,05$ & $82 / 18$ \\
$\begin{array}{l}\text { Incisión quirúrgica } \\
\text { benigna (\%) (IQB) }\end{array}$ & 29,9 & 15 & 28,6 & $\mathrm{p}>0,05$ & 25,2 \\
$\begin{array}{l}\text { Incisión quirúrgica } \\
\text { maligna (\%) (IQM) }\end{array}$ & 13,2 & 25 & 11,8 & $\mathrm{p}>0,05$ & 19 \\
\begin{tabular}{l} 
Márgenes positivos (\%) \\
\hline
\end{tabular} & 5,8 & 15,4 & 0 & $\mathrm{p}>0,05$ & 5,7 \\
\hline
\end{tabular}

mejor manera es mediante la figura de un tutor. Heniford et al. demostraron que el aprendizaje de la esplenectomía laparoscópica era más efectiva mediante un tutor y que la aparición de avances en la técnica, dependía de la experiencia previa de la misma ${ }^{4}$. Asimismo, se ha demostrado que los programas de formación intensivos reducen las tasas de complicaciones ${ }^{5}$.

Fabricio et al, propusieron el modelo con tutor mediante los tres grupos según los cirujanos ${ }^{6}$. No observaron diferencias significativas entre los tres tiempos quirúrgicos de la prostatectomía radical debido quizás a un reducido número de casos (Grupo 1: 12, 2: 18, 3: 20). La presencia del tutor evita los pasos y movimientos innecesarios durante la cirugía y también la posibilidad de reconversión a cirugía abierta si el alumno se encuentra "atascado" en un determinado paso, garantizando la continuidad del proceso. Estos momentos difíciles pueden ser: la localización del cuello, el acceso al plano vesicular, el control del recto, la hemostasia del Santorini, y la sutura uretro-vesical.

En el Grupo 1 (Tutor + Alumno) observamos una reducción significativa del tiempo quirúrgico, debido probablemente a varios factores a parte de la propia curva de aprendizaje del tutor:

- El inicio desde cero de un programa de cirugía laparoscópica, con el adiestramiento de enfermería y anestesiología, disponibilidad del material/instrumental adecuado etc.

- La curva de aprendizaje del propio ayudantealumno.

- La incorporación de la vía extraperitoneal como variante (en los últimos 20 casos).

Por otro lado llama la atención que en los grupos 2 y 3 la media de tiempo quirúrgico sea similar. Nuestra interpretación es que esto es reflejo de una buena docencia en la sistemática de los pasos quirúrgicos, que son fácilmente reproducibles sin la figura del tutor en el tercer grupo. Este hecho se podría considerar como herramienta para evaluar el control de calidad de un programa de aprendizaje con tutor como el que proponemos. 
Por otro lado, aparte de un aumento del 25\% del tiempo quirúrgico, no se observa ningún otro "peaje" sobre el paciente cuando el primer cirujano es el alumno. Por tanto este sistema además de ser efectivo puede considerarse seguro.

Existe el debate sobre el número necesario de procedimientos para completar la curva de aprendizaje, habiéndose estimado entre 40-100 $\operatorname{casos}^{7}$, aunque parece que con este esquema de aprendizaje el número sería menor.

Creemos que la prostatectomía radical laparoscópica es una técnica en constante evolución, mejorable día a día y con objetivos que van apareciendo a medida que se consolidan los pasos estándares del procedimiento. Estos nuevos objetivos pueden ser, por ejemplo, el control de los haces neurovasculares y del cuello vesical, para unos mejores resultados en la continencia y potencia. Es por tanto el afinamiento de esta cirugía, en detrimento del tiempo quirúrgico, lo que la hace más valiosa, competitiva y futura, frente a otras opciones de tratamiento del cáncer de próstata clínicamente órgano confinado.

Otro ejercicio muy interesante y complementario en el aprendizaje podría ser el propuesto por Touijer et al donde analizan los videos de las cirugías y lo correlacionan con el resultado anátomo-patológico desde ell punto de vista de los márgenes. Observan que esta revisión quirúrgica-patológica reduce el porcentaje de invasiones capsulares ${ }^{8}$.

\section{CONCLUSIONES}

Nuestro método de aprendizaje está formando de manera segura y efectiva a esta segunda generación de urólogos en la ejecución de una prostatectomía radical laparoscópica, y de un modo más rápido que en los trabajos hasta ahora publicados. La presencia de un laparoscopista experto como tutor le da a este método la agilidad y la experiencia necesarias para reducir el tiempo quirúrgico y de incorporación de nuevos cirujanos. En espera de los resultados funcionales y oncológicos a largo plazo, este sistema de aprendizaje no se asocia ni con un mayor riesgo para el paciente ni con un peor control quirúrgico/oncológico.

\section{REFERENCIAS}

1. Guillonneau B, Vallancien G.Laparoscopic radical prostatectomy: the Montsouris technique. J Urol. 2000,163(6): 1643-1649.

2. Hasson HM. Laparoscopic cannula cone with means for cannula stabilization and wound closure. J Am Assoc Gynecol Laparosc. 1998;5(2):183-185.

3. Bollens R, Sandhu S, Roumeguere T, Quackels T, Schulman C. Laparoscopic radical prostatectomy: the learning curve. Curr Opin Urol. 2005;15(2):79-82.

4. Heniford BT, Backus CL, Matthews BD, Greene FL, Teel WB, Sing RF. Optimal teaching environment for laparoscopic splenectomy. Am J Surg. 2001;181(3):226-230.

5. Cadeddu JA, Wolfe JS Jr, Nakada S, Chen R, Shalhav A, Bishoff JT, et al. Complications of laparoscopic procedures after concentrated training in urological laparoscopy. J Urol. 2001;166(6):2109-2111.

6. Fabrizio MD, Tuerk I, Schellhammer PF. Laparoscopic radical prostatectomy: decreasing the learning curve using a mentor initiated approach. J Urol. 2003;169(6):2063-2065.

7. Martina GR, Giumelli P, Scuzzarella S, Remotti M, Caruso G, Lovisolo J. Laparoscopic extraperitoneal radical prostatectomy-learning curve of a laparoscopy naive urologist in a community hospital. Urology. 2005;65(5):959-963.

8. Touijer K, Kuroiwa K, Saranchuk JW, Hassen WA, Trabulsi EJ, Reuter VE,et al. Quality improvement in laparoscopic radical prostatectomy for pT2 prostate cancer: impact of video documentation review on positive surgical margin. $\mathrm{J}$ Urol. 2005;173(3):765-768.

Correspondencia autor: Dr. C.X. Raventós Busquets Servei d'Urologia. Hospital Univ. Vall d'Hebron.

Passeig de la Vall D’Hebrón, 119-129. 08035 Barcelona. Tel.: 932746000

E-mail: cravento@vhebron.net

Información artículo: Original - Laparoscopia

Trabajo recibido: septiembre 2006

Trabajo aceptado: enero 2007 